\title{
Personality-Related Determinants of Subtle Cognitive Decline in Old Age: A Population-Based Study
}

\author{
Cristelle Rodriguez $^{a} \quad$ Emiliano Albanese ${ }^{a} \quad$ Alan Pegna $^{c}$ Simona Toma ${ }^{a}$ \\ Marine Ackermann $^{a} \quad$ Eline Tombeur $^{a} \quad$ Francois R. Herrmann ${ }^{b}$ \\ Panteleimon Giannakopoulos ${ }^{a}$ \\ a Division of General Psychiatry, Department of Mental Health and Psychiatry, ${ }^{b}$ Division \\ of Geriatrics, Department of Internal Medicine, Rehabilitation and Geriatrics, and \\ 'Neuropsychology Unit and Department of Neurology, Geneva University Hospitals and \\ University of Geneva School of Medicine, Geneva, Switzerland
}

\section{Key Words}

Personality · NEO-Personality Inventory-Revised · Elderly controls · Cognitive decline · Mild cognitive impairment $\cdot$ Openness

\begin{abstract}
Background/Aims: Recent studies of cases with mild cognitive impairment (MCI) suggested that besides Alzheimer disease $(A D)$-related biomarkers, some personality dimensions are associated with progression to AD. To date, there are no studies addressing the psychological determinants of subtle cognitive decline in healthy elderly controls. Methods: 488 community-dwelling healthy controls were assessed with a detailed neuropsychological battery at baseline and an 18-month follow-up. Personality factors and facets were investigated at baseline using the NEO-Personality Inventory-Revised (NEO-PI-R). Upon follow-up, there were 264 stable controls $(\mathrm{sCON})$ and 224 deteriorating controls (dCON). Their personality data were compared to those of the $102 \mathrm{MCI}$ cases using one-way analysis of variance and logistic regression models. Results: Significantly higher scores of Openness factor (as well as Aesthetics, Ideas and Values facets) were found in SCON than in both dCON and MCI cases. The three groups did not differ in the other NEO-PI-R factor and facet scores. Openess factor (and the same facets) was associated with cognitive preservation in healthy controls (OR: $0.72,95 \% \mathrm{CI}$ : $0.59,0.87)$. Lower scores in the same factor and facets conferred higher risk to have MCI (OR: $0.61,95 \%$ CI: $0.46,0.79)$. Conclusion: Higher openness to new experiences and thoughts may be a protective factor against early cognitive decline in brain aging.
\end{abstract}




\section{Introduction}

Subtle decrease in episodic memory and executive function performances typically occurs in the course of normal aging [1]. Most of these decreases are benign, remain stable over time and do not affect daily living and quality of life. In some cases, cognitive deficits may rapidly progress to pre-mild cognitive impairment (MCI) and MCI states. These constructs correspond to the transitional state between normal cognitive aging and dementia and are based on the presence of subjective concern for and an objective evidence of cognitive decline with the overall preservation of independence and only mild problems with complex functional abilities [2,3]. Preceding the pre-MCI state, the concept of preclinical Alzheimer disease (AD) was introduced designing elderly controls with progressive installation of clinically silent AD-related molecular and imaging changes [4]. These persons who usually display PiBpositive deposits in positron emission tomography scans and decreased concentration of A 342 in cerebrospinal fluid (CSF) remain cognitively stable for variable time periods but ultimately display significantly increased risk for AD [5]. Stressing the difficulty to isolate cases at high risk of $\mathrm{AD}$ among these persons, a 5-year follow-up showed that more than $60 \%$ among them have a stable memory trajectory, $36 \%$ display subtle memory decline and only $4 \%$ progress to pre-MCI [6]. Markers of $\mathrm{A} \beta$ accumulation (decreased levels of CSF A $\beta 42$, positive positron emission tomography amyloid imaging) and neurodegeneration (increased CSF t-tau and p-tau, increased hippocampal atrophy or cortical thinning) as well as functional magnetic resonance imaging and electroencephalographic abnormalities have been shown to be associated with increased rates of cognitive decline in this group [7-10].

Unlike AD-related biomarkers, data regarding the psychological determinants of subtle cognitive deterioration in healthy elders are still scarce $[11,12]$. Among them, personality traits have been drawing growing interest since they are associated with MCI status and may modulate independently dementia risk in MCI [13-15]. According to a widely accepted taxonomic approach, there are five major personality traits (i.e. Neuroticism, Extraversion, Openness, Agreeableness and Conscientiousness) that are typically measured with the Neuroticism Extraversion Openness Personality Inventory-Revised (NEO-PI-R), a crossculturally validated instrument $[16,17]$. A few longitudinal studies explored the predictive role of personality factors in AD. Overall, high levels of Neuroticism increase, whereas high levels of Conscientiousness and Openness decrease the dementia risk in MCI cases [18]. To date, there is no longitudinal study addressing this issue in elderly controls.

Using data from a large, population-based study of community-dwelling older adults who have undergone an 18-month follow-up, we aimed to explore whether personality factors and facets were associated with rapid changes in cognitive function before the emergence of MCI. Because sociodemographic characteristics (i.e. age and educational level) [19] and anxiety and depressive symptomatology may influence cognitive performance and decline [20] these were also accounted for in the present analysis.

\section{Materials and Methods}

\section{Study Protocol and Participants}

Community-dwelling men and women aged 50 years and older, living in the Frenchspeaking part of Switzerland were censed and invited to take part into a prospective cohort study designed to investigate the determinants and trajectories of cognitive function in late life. A telephone screening interview was used to exclude those with self-reported major physical and mental illnesses (including cancer, severe visual impairment, psychiatric and neurologic disorders and alcohol or drug abuse), and with a history of cerebro- and cardio- 
vascular diseases. Those under neuro-psychopharmacological treatment (including narcoleptics, antidepressants and psychostimulants) were excluded. Eligible participants were assessed by board-certified neuropsychologists under the supervision of a senior psychiatrist. Data on age (years), sex and educational level (primary, less than 9 years; secondary, between 9 and 12 years; and tertiary or university level, more than 12 years) were collected at the first visit. All participants provided written informed consent, and standard approval of the study was obtained from the local Ethics Committee of the University Hospitals of Geneva.

\section{Neuropsychological Assessment}

Participants were evaluated at inclusion with an extensive neuropsychological battery, including the Mini-Mental State Examination (MMSE) [21], the Hospital Anxiety and Depression Scale (HAD) [22], and the Lawton Instrumental Activities of Daily Living (IADL) [23]. Cognitive assessment included (a) attention (Digit-Symbol-Coding [24], Trail Making Test A [25]); (b) working memory [verbal: Digit Span Forward [24], visuospatial: Visual Memory Span (Corsi) [24]]; (c) episodic memory (verbal: RI-48 Cued Recall Test [26] or RL/ RI-16 Free and Cued Recall Test [27], visual: Shapes Test [28]); (d) executive functions [Trail Making Test B [25], Wisconsin Card Sorting Test (WCST) [29] and Phonemic Verbal Fluency Test [30]]; (e) language (Boston Naming Test [31]); (f) visual gnosis (Ghent Overlapping Figures [32]), and (g) praxis [ideomotor [33], reflexive [34], and constructional (Consortium to Establish a Registry for Alzheimer's Disease, CERAD), figures copy [35]]. All individuals were also evaluated with the Clinical Dementia Rating (CDR) scale [36].

Those who met dementia DSM-IV diagnostic criteria on the basis of the neuropsychological and clinical assessments were excluded. In agreement with the criteria of Petersen et al. [37], participants with a CDR score of 0.5 but no dementia and a score more than 1.5 standard deviations (SD) below the age-appropriate mean in any of the previously mentioned tests were confirmed to have MCI. Participants with neither dementia nor MCI were classified as cognitively healthy older 'controls' and underwent a full neuropsychological assessment at follow-up, on average 18 months later. Participants whose cognitive scores remained stable were classified as stable controls (sCON), and those whose performance at follow-up was at least $0.5 \mathrm{SD}$ lower compared to the first evaluation, in at least two cognitive tests, were classified as deteriorating ( $\mathrm{dCON}$ ) controls. All individuals were clinically assessed independently by two trained neuropsychologists (S.T. and M.A.). The final classification of dCON was made blindly by a board-certified psychologist (C.R., 10 years of experience) who took into account both the neuropsychological scores and clinical assessment [38].

The final sample included 590 participants, of which 264 were classified as sCON [age 71.56 (4.35 SD) years; 160 women], $224 \mathrm{dCON}$ [age 73.12 (4.82 SD) years; 137 women], and 102 met MCI diagnostic criteria [age 71.78 (6.06 SD) years; 37 women].

\section{Personality Assessment}

Personality features and dimensions were assessed at baseline using the French version of the NEO-PI-R $[16,17]$. Participants were asked to complete the 240 -item self-report version of the NEO-PI-R questionnaire using a five-point Likert agreement scale. The NEO-PI-R assesses 30 facets, 6 for each of the following five personality factors: Neuroticism, which is the tendency to feel negative affect including anxiety, hostility and anger; Extraversion, which encapsulates the proneness towards positive emotions and feelings such as warmth and enthusiasm; Openness, which is the personal inclination to experience and the appreciation of new situations and thoughts with a curious, imaginative and creative attitude, which is defined along six facets that cover imagination (or fantasy), sense of aesthetics, emotions and feelings, but also proactive behaviors and actions to explore and experiment beyond habits 
Rodriguez et al.: Personality-Related Determinants of Subtle Cognitive Decline in Old Age: A Population-Based Study

and routines, as well as intellectual curiosity, and the disposition to negotiate and discuss social, political and religious values; Agreeableness, which is characterized by trustful, cooperative and altruistic tendencies, and finally Consciousness, which is the predisposition to be reliable, resolute and well organized, and unwilling to deviate from rules and moral principles.

\section{Statistical Analysis}

Continuous variables at baseline were compared among the 3 groups using one-way ANOVA and categorical variables using the Kruskal-Wallis test. Pairwise comparisons were performed after Bonferroni's correction. Gender differences were assessed using the $\chi^{2}$ test. The comparison of continuous variables between two groups (neuropsychological differences between sCON and dCON at follow-up), Neuroticism factors and its facets versus a reference population [39] were performed using the unpaired t test.

To assess the discriminating values of personality factors and facets, we included them, after a z-score transform, as independent variables in univariate and adjusted (for age, education and sex) logistic regression models to predict either the distinction between sCON/ dCON or that between sCON/MCI. Z-scores were computed with mean and SD for each factor and facet using the sCON group as the reference population. The coefficient of determination is presented as pseudo- $\mathrm{R}^{2}$. All analyses have been conducted using STATA 14.0 (STATA Corp., College Station, Tex., USA, 2015)

\section{Results}

Demographic and neuropsychological data in the three diagnostic groups at baseline are presented in table 1. Regarding demographic factors, dCON were older than $\mathrm{sCON}(\mathrm{p}=$ $0.001)$. More men were present in the MCI groups ( $<<0.001)$, and the sCON counted less depressive participants than the MCI group ( $p=0.039)$. The educational level was similar among the three groups $(\mathrm{p}=0.157)$. There were no significant differences in episodic memory cognitive performances between sCON and dCON at baseline. Slight but still significant group differences were found in Digit-Symbol-Coding $(\mathrm{p}<0.001)$, Shapes Test immediate cued recall $(p<0.001)$ and praxis ideomotor test $(p=0.007)$ scores. As expected, significant differences were found at baseline between SCON and MCI as well as between dCON and MCI in MMSE ( $\mathrm{p}<0.001$ ), RI-48 (total immediate cued recall, total cued recall, intrusions; $p<0.001)$, Verbal Fluency $(\mathrm{p}<0.001)$, Trail Making Test $\mathrm{B}$ time $(\mathrm{p}<0.001)$, Trail Making Test B/A $(p<0.001)$, and Shapes Test (total score immediate three recall and delayed recall; $\mathrm{p}<0.001$ ) scores.

In respect to personality, only Openness factor scores differed between sCON and the two other groups [Openness, $F(2,587)=14.71, \mathrm{p}<0.001$; table 2]. This also concerned the Openness facets of Aesthetics $(02)[F(2,587)=6.49, \mathrm{p}=0.002]$, Ideas $[F(2,587)=9.12, \mathrm{p}<$ $0.001]$, and Values $(05)[F(2,587)=11.82, \mathrm{p}<0.001]$. Significant differences were evident between the sCON and the MCI group in the Action $(04),[F(2,587)=10.81, \mathrm{p}<0.001]$ as well as in the Fantasy facet $(01),[F(2,587)=4.86, p=0.008]$ scores. In addition, sCON showed significantly higher scores in the Deliberation facet (C5) compared to the MCI group $[F(2$, $587)=4.65, p=0.010]$. Significant differences were also found between the dCON and the MCI group for Gregariousness (E2) $[F(2,587)=3.45, \mathrm{p}=0.033]$, Excitement seeking $(E 5)[F(2$, $587)=4.78, \mathrm{p}=0.009]$ and Action $(04)[F(2,587)=10.81, \mathrm{p}<0.001]$.

At follow-up, sCON had significantly better performances than dCON in all of the neuropsychological functions except visual gnosis (table 3). 
Table 1. Demographic and neuropsychological data in the three diagnostic groups at baseline (sCON dCON and $\mathrm{MCI}$ )

\begin{tabular}{|c|c|c|c|c|}
\hline & $\begin{array}{l}\text { sCON } \\
(n=264)\end{array}$ & $\begin{array}{l}\mathrm{dCON} \\
(\mathrm{n}=224)\end{array}$ & $\begin{array}{l}\text { MCI } \\
(n=102)\end{array}$ & $\mathrm{p}$ \\
\hline Female gender, $\%$ & $160 \pm 60.61$ & $137 \pm 61.16$ & $37 \pm 36.27$ & $<0.001^{\mathrm{b}, \mathrm{c}}$ \\
\hline Age, years & $71.56 \pm 4.35$ & $73.12 \pm 4.82$ & $71.78 \pm 6.06$ & $0.001^{\mathrm{a}}$ \\
\hline Education, \% & & & & 0.157 \\
\hline$<9$ years & $40 \pm 47.62$ & $31 \pm 36.90$ & $13 \pm 15.48$ & \\
\hline $9-12$ years & $107 \pm 40.53$ & $118 \pm 52.68$ & $45 \pm 44.12$ & \\
\hline$>12$ years & $117 \pm 44.32$ & $75 \pm 33.48$ & $236 \pm 40.00$ & \\
\hline MMSE & $28.51 \pm 1.23$ & $28.43 \pm 1.49$ & $27.38 \pm 1.85$ & $<0.001^{\mathrm{b}, \mathrm{c}}$ \\
\hline IADL & $8.29 \pm 0.78$ & $8.25 \pm 0.94$ & $8.48 \pm 1.02$ & 0.167 \\
\hline HAD Total & $5.55 \pm 3.26$ & $5.85 \pm 3.70$ & $5.91 \pm 3.63$ & 0.535 \\
\hline Anxiety & $4.23 \pm 2.47$ & $4.28 \pm 2.72$ & $4.12 \pm 2.57$ & 0.877 \\
\hline Depression & $1.34 \pm 1.47$ & $1.58 \pm 1.71$ & $1.79 \pm 1.76$ & $0.039^{b}$ \\
\hline Digit Span Forward & $5.62 \pm 1.13$ & $5.81 \pm 1.00$ & $5.55 \pm 0.99$ & 0.054 \\
\hline $\begin{array}{l}\text { Visual Memory Span Forward } \\
\text { RI-48 Cued Recall Test }\end{array}$ & $5.18 \pm 1.00$ & $5.14 \pm 0.93$ & $5.14 \pm 1.02$ & 0.892 \\
\hline Immediate verbal cued recall & $40.05 \pm 4.43$ & $39.53 \pm 4.88$ & $35.69 \pm 5.20$ & $<0.001^{\mathrm{b}, \mathrm{c}}$ \\
\hline Delayed cued recall & $27.69 \pm 5.02$ & $27.20 \pm 5.26$ & $16.94 \pm 4.96$ & $<0.001^{\mathrm{b}, \mathrm{c}}$ \\
\hline Intrusions & $2.25 \pm 2.53$ & $2.20 \pm 2.36$ & $4.74 \pm 5.53$ & $<\mathbf{0 . 0 0 1} 1^{\mathrm{b}, \mathrm{c}}$ \\
\hline \multicolumn{5}{|l|}{ Shapes Test } \\
\hline Total score (3 immediate recalls) & $34.00 \pm 3.67$ & $32.91 \pm 4.49$ & $29.95 \pm 7.20$ & $<0.001^{\mathrm{a}, \mathrm{b}, \mathrm{c}}$ \\
\hline Delayed recall & $11.70 \pm 0.71$ & $11.46 \pm 1.19$ & $10.16 \pm 2.74$ & $<0.001^{\mathrm{b}, \mathrm{c}}$ \\
\hline Boston Naming Test & $19.36 \pm 0.95$ & $19.30 \pm 1.76$ & $19.72 \pm 3.25$ & 0.214 \\
\hline Digit-Symbol-Coding & $56.78 \pm 11.73$ & $51.60 \pm 11.40$ & $49.50 \pm 10.80$ & $<0.001^{\mathrm{a}, \mathrm{b}}$ \\
\hline \multicolumn{5}{|l|}{ Trail Making Test A } \\
\hline Time, $\mathrm{s}$ & $39.70 \pm 14.00$ & $41.78 \pm 12.35$ & $43.39 \pm 13.86$ & $0.040^{b}$ \\
\hline Error & $0.08 \pm 0.28$ & $0.08 \pm 0.31$ & $0.07 \pm 0.36$ & 0.907 \\
\hline \multicolumn{5}{|l|}{ Trail Making Test B } \\
\hline Time, s & $94.59 \pm 40.87$ & $103.43 \pm 40.22$ & $126.67 \pm 67.94$ & $<\mathbf{0 . 0 0 1} 1^{\mathrm{b}, \mathrm{c}}$ \\
\hline Error & $0.47 \pm 0.76$ & $0.49 \pm 0.72$ & $0.65 \pm 1.04$ & 0.163 \\
\hline Trail Making Test B/A & $2.48 \pm 0.91$ & $2.58 \pm 0.99$ & $2.99 \pm 1.31$ & $<0.001^{\mathrm{b}, \mathrm{c}}$ \\
\hline Verbal Fluency & $22.15 \pm 5.83$ & $22.18 \pm 6.29$ & $19.35 \pm 7.05$ & $<0.001^{\mathrm{b}, \mathrm{c}}$ \\
\hline \multicolumn{5}{|l|}{ WCST } \\
\hline Categories completed & $4.74 \pm 2.08$ & $4.32 \pm 2.21$ & $3.97 \pm 2.37$ & $0.036^{b}$ \\
\hline Failure to maintain set & $0.14 \pm 0.37$ & $0.14 \pm 0.39$ & $0.20 \pm 0.51$ & 0.521 \\
\hline \multicolumn{5}{|l|}{ Praxis } \\
\hline Constructional (CERAD) & $10.82 \pm 0.58$ & $10.73 \pm 0.72$ & $10.73 \pm 0.76$ & 0.245 \\
\hline Ideomotor transitive & $9.44 \pm 0.75$ & $9.24 \pm 1.02$ & $9.09 \pm 1.18$ & $0.007^{\mathrm{a}, \mathrm{b}}$ \\
\hline Ideomotor intransitive & $19.55 \pm 0.94$ & $19.42 \pm 0.99$ & $19.45 \pm 0.87$ & 0.338 \\
\hline Reflexive & $7.06 \pm 1.03$ & $6.83 \pm 1.15$ & $6.91 \pm 1.19$ & 0.074 \\
\hline Visual gnosis (Ghent) & $4.99 \pm 0.09$ & $5.00 \pm 0.07$ & $4.97 \pm 0.18$ & 0.129 \\
\hline
\end{tabular}

Values are presented as mean \pm SD unless otherwise indicated.

a Significant difference between sCON and dCON. ${ }^{b}$ Significant difference between sCON and MCI. ${ }^{c}$ Significant difference between dCON and MCI. 
Table 2. Personality factor and facet values in the three diagnostic groups at baseline

\begin{tabular}{|c|c|c|c|c|c|}
\hline Personality traits & & sCON & $\mathrm{dCON}$ & MCI & $\mathrm{p}$ \\
\hline Neuroticism & $\mathrm{N}$ & $76.34 \pm 18.82$ & $76.71 \pm 18.24$ & $77.30 \pm 18.98$ & 0.904 \\
\hline Anxiety & $\mathrm{N} 1$ & $13.59 \pm 5.29$ & $13.88 \pm 5.14$ & $14.28 \pm 5.30$ & 0.513 \\
\hline Angry hostility & $\mathrm{N} 2$ & $11.93 \pm 4.07$ & $12.07 \pm 3.98$ & $12.51 \pm 4.24$ & 0.471 \\
\hline Depression & N3 & $12.53 \pm 4.68$ & $13.10 \pm 4.39$ & $12.59 \pm 4.40$ & 0.345 \\
\hline Self-consciousness & $\mathrm{N} 4$ & $14.00 \pm 4.15$ & $13.70 \pm 3.95$ & $13.59 \pm 3.96$ & 0.580 \\
\hline Impulsiveness & N5 & $14.45 \pm 4.23$ & $13.96 \pm 3.61$ & $13.70 \pm 3.58$ & 0.174 \\
\hline Vulnerability & N6 & $9.83 \pm 3.68$ & $10.00 \pm 4.27$ & $10.12 \pm 4.54$ & 0.812 \\
\hline Extraversion & $\mathrm{E}$ & $102.34 \pm 16.54$ & $100.06 \pm 17.12$ & $102.47 \pm 17.15$ & 0.268 \\
\hline Warmth & E1 & $22.72 \pm 4.25$ & $22.42 \pm 4.46$ & $22.91 \pm 4.05$ & 0.577 \\
\hline Gregariousness & E2 & $14.17 \pm 5.07$ & $13.62 \pm 4.93$ & $15.18 \pm 4.87$ & $0.033^{c}$ \\
\hline Assertiveness & E3 & $15.07 \pm 4.19$ & $14.93 \pm 4.61$ & $14.81 \pm 4.64$ & 0.869 \\
\hline Activity & $\mathrm{E} 4$ & $18.04 \pm 4.34$ & $17.87 \pm 4.55$ & $17.10 \pm 4.31$ & 0.182 \\
\hline Excitement seeking & E5 & $12.70 \pm 4.07$ & $12.31 \pm 4.06$ & $13.80 \pm 4.05$ & $0.009^{c}$ \\
\hline Positive emotion & E6 & $19.65 \pm 4.66$ & $18.92 \pm 4.82$ & $18.66 \pm 4.81$ & 0.105 \\
\hline Openness & 0 & $115.15 \pm 17.80$ & $108.86 \pm 17.39$ & $105.31 \pm 16.10$ & $<\mathbf{0 . 0 0 1}^{\mathrm{a}, \mathrm{b}}$ \\
\hline Fantasy & 01 & $17.99 \pm 4.99$ & $17.24 \pm 4.14$ & $16.38 \pm 4.30$ & $0.008^{\mathrm{b}}$ \\
\hline Aesthetics & 02 & $19.47 \pm 5.26$ & $18.13 \pm 5.31$ & $17.60 \pm 4.87$ & $0.002^{a, b}$ \\
\hline Feelings & 03 & $19.64 \pm 4.18$ & $18.80 \pm 3.90$ & $18.75 \pm 3.96$ & 0.039 \\
\hline Actions & 04 & $16.80 \pm 3.97$ & $15.98 \pm 3.83$ & $14.73 \pm 3.71$ & $<0.001^{\text {b,c }}$ \\
\hline Ideas & 05 & $20.64 \pm 4.33$ & $19.25 \pm 4.97$ & $18.69 \pm 4.17$ & $<0.001^{\mathrm{a}, \mathrm{b}}$ \\
\hline Values & 06 & $20.61 \pm 3.38$ & $19.46 \pm 3.40$ & $18.94 \pm 3.28$ & $<0.001^{\mathrm{a}, \mathrm{b}}$ \\
\hline Agreeableness & $\mathrm{A}$ & $132.27 \pm 15.04$ & $130.41 \pm 16.75$ & $129.52 \pm 15.41$ & 0.233 \\
\hline Trust & A1 & $21.33 \pm 3.93$ & $20.65 \pm 4.17$ & $21.00 \pm 3.73$ & 0.171 \\
\hline Straightforwardness & A2 & $23.28 \pm 4.26$ & $23.21 \pm 4.38$ & $22.68 \pm 4.49$ & 0.470 \\
\hline Altruism & A3 & $23.64 \pm 3.29$ & $23.35 \pm 3.44$ & $23.45 \pm 3.34$ & 0.634 \\
\hline Compliance & $\mathrm{A} 4$ & $20.59 \pm 3.99$ & $20.42 \pm 4.09$ & $19.89 \pm 3.91$ & 0.324 \\
\hline Modesty & A5 & $21.31 \pm 4.19$ & $21.31 \pm 4.43$ & $21.44 \pm 3.87$ & 0.961 \\
\hline Tender-mindedness & A6 & $22.11 \pm 3.47$ & $21.45 \pm 3.87$ & $21.14 \pm 3.72$ & 0.036 \\
\hline Conscientiousness & $\mathrm{C}$ & $120.36 \pm 17.33$ & $122.32 \pm 16.6$ & $122.46 \pm 22.24$ & 0.406 \\
\hline Competence & $\mathrm{C} 1$ & $20.55 \pm 3.28$ & $20.25 \pm 3.59$ & $20.33 \pm 3.37$ & 0.607 \\
\hline Order & $\mathrm{C} 2$ & $18.25 \pm 4.28$ & $18.58 \pm 4.47$ & $19.16 \pm 4.24$ & 0.195 \\
\hline Dutifulness & $\mathrm{C} 3$ & $24.77 \pm 3.38$ & $24.94 \pm 3.19$ & $24.73 \pm 4.15$ & 0.819 \\
\hline Achievement striving & $\mathrm{C} 4$ & $18.69 \pm 3.71$ & $19.29 \pm 3.50$ & $19.52 \pm 3.33$ & 0.067 \\
\hline Self-discipline & $\mathrm{C} 5$ & $19.88 \pm 4.50$ & $20.32 \pm 4.55$ & $20.59 \pm 4.67$ & 0.335 \\
\hline Deliberation & $\mathrm{C} 6$ & $18.22 \pm 4.17$ & $18.94 \pm 3.73$ & $19.65 \pm 5.08$ & $0.010^{b}$ \\
\hline
\end{tabular}

Values are presented as mean \pm SD

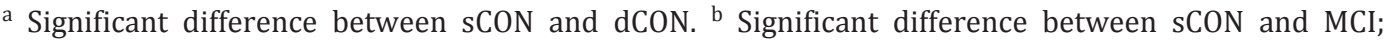
c Significant difference between dCON and MCI.

Regression data on the association between NEO-PI factors and facets (independent variables) and the risk to experience subtle cognitive deterioration (dCON) as compared to sCON are summarized in table 4 . Only Openness was significantly associated with a $42.8 \%$ $(1.428=1 / 0.70 ; \mathrm{OR}=0.70 ; 95 \% \mathrm{CI} 0.58-0.84)$ risk reduction of being in the $\mathrm{dCON}$ group for $1 \mathrm{z}$-score of this factor. After adjusting for age, sex and educational level $\left(\mathrm{OR}_{\text {adjusted }}=0.72\right.$; $95 \%$ CI $0.59-0.87$ ), this factor explained almost $5 \%$ of the sCON/dCON variance. Amongst the Openness facets, Aesthetics, Feelings, Ideas and Values are always significantly reducing the risk of deterioration, with and without adjustment, with $\mathrm{OR}_{\text {adjusted }}$ ranging from 0.76 to 0.81 (table 4). 
Dementia

and Geriatric

Cognitive Disorders

Table 3. Neuropsychological differences between $\mathrm{SCON}$ and dCON at follow-up

\begin{tabular}{l|l}
\hline Dement Geriatr Cogn Disord Extra 2016;6:120-132 \\
\hline DOI: 10.1159/000441388 & $\begin{array}{l}\text { (c) 2016 S. Karger AG, Basel } \\
\text { www.karger.com/dee }\end{array}$ \\
\hline
\end{tabular}

Rodriguez et al.: Personality-Related Determinants of Subtle Cognitive Decline in Old Age: A Population-Based Study

\begin{tabular}{|c|c|c|c|}
\hline & $\begin{array}{l}\text { sCON } \\
(n=264)\end{array}$ & $\begin{array}{l}\mathrm{dCON} \\
(\mathrm{n}=224)\end{array}$ & $\begin{array}{l}\mathrm{p} \\
\text { (t test) }\end{array}$ \\
\hline Age, years & $71.56 \pm 4.35$ & $73.12 \pm 4.82$ & $<0.001$ \\
\hline MMSE & $28.59 \pm 1.18$ & $28.05 \pm 1.50$ & $<0.001$ \\
\hline IADL & $8.19 \pm 0.58$ & $8.29 \pm 0.88$ & 0.147 \\
\hline HAD Total & $5.77 \pm 3.45$ & $6.17 \pm 3.96$ & 0.228 \\
\hline Anxiety & $4.24 \pm 2.64$ & $4.51 \pm 3.03$ & 0.299 \\
\hline Depression & $1.53 \pm 1.48$ & $1.67 \pm 1.75$ & 0.350 \\
\hline Digit Span Forward & $5.85 \pm 1.06$ & $5.53 \pm 1.03$ & 0.001 \\
\hline $\begin{array}{l}\text { Visual Memory Span Forward } \\
\text { RI-48 Cued Recall Test }\end{array}$ & \multicolumn{2}{|c|}{ RI-48 Cued Recall Test } & $<0.001$ \\
\hline Immediate verbal cued recall & $41.11 \pm 4.49$ & $39.31 \pm 0.96$ & $<0.001$ \\
\hline Delayed cued recall & $29.66 \pm 29.66$ & $26.17 \pm 6.19$ & $<0.001$ \\
\hline Intrusions & $2.11 \pm 2.53$ & $3.40 \pm 3.32$ & $<0.001$ \\
\hline \multicolumn{4}{|l|}{ Shapes test } \\
\hline \multicolumn{4}{|l|}{ Total score } \\
\hline (3 immediate recalls) & $34.46 \pm 3.03$ & $32.98 \pm 4.17$ & $<0.001$ \\
\hline Delayed recall & $11.83 \pm 1.75$ & $11.13 \pm 1.54$ & $<0.001$ \\
\hline Boston Naming Test & $19.68 \pm 1.99$ & $19.16 \pm 1.92$ & 0.003 \\
\hline Digit-Symbol-Coding & $56.61 \pm 11.64$ & $51.25 \pm 11.89$ & $<0.001$ \\
\hline \multicolumn{4}{|l|}{ Trail Making Test A } \\
\hline Time, s & $39.28 \pm 12.86$ & $43.42 \pm 12.92$ & 0.001 \\
\hline Error & $0.07 \pm 0.29$ & $0.13 \pm 0.42$ & 0.083 \\
\hline \multicolumn{4}{|l|}{ Trail Making Test B } \\
\hline Time, s & $86.08 \pm 33.17$ & $115.26 \pm 47.71$ & $<0.001$ \\
\hline Error & $0.21 \pm 0.59$ & $0.70 \pm 0.95$ & $<0.001$ \\
\hline Trail Making Test B/A & $2.27 \pm 0.78$ & $2.73 \pm 0.98$ & $<0.001$ \\
\hline Verbal Fluency & $21.25 \pm 6.01$ & $18.82 \pm 6.08$ & $<0.001$ \\
\hline \multicolumn{4}{|l|}{ WCST } \\
\hline Categories completed & $5.04 \pm 1.87$ & $4.00 \pm 2.33$ & $<0.001$ \\
\hline Failure to maintain set & $0.13 \pm 0.41$ & $0.28 \pm 0.57$ & 0.001 \\
\hline Praxis & $10.84 \pm 0.49$ & $10.70 \pm 0.72$ & 0.015 \\
\hline Constructional (CERAD) & & & \\
\hline Ideomotor transitive & $9.66 \pm 0.71$ & $9.46 \pm 0.82$ & 0.003 \\
\hline Ideomotor intransitive & $19.66 \pm 0.79$ & $19.33 \pm 0.97$ & $<0.001$ \\
\hline Reflexive & $7.37 \pm 0.87$ & $7.15 \pm 1.06$ & 0.012 \\
\hline Visual gnosis (Ghent) & $5.00 \pm 0.00$ & $5.00 \pm 0.00$ & 0.318 \\
\hline
\end{tabular}

Values are presented as mean \pm SD.

The associations of personality factors and facets with the risk of being in the MCI group as compared to sCON are shown in table 5. Only Openness was significantly associated with a $65.0 \%\left(1.650=1 / 0.606 ; \mathrm{OR}_{\text {adjusted }}=0.61 ; 95 \% \mathrm{CI} 0.46-0.79\right)$ risk reduction of being in the $\mathrm{MCI}$ group for $1 \mathrm{z}$-score of this factor. The adjusted regression model explained $8 \%$ of the sCON/MCI variance. All but one of the Openness facets showed similar associations and effect sizes (table 5).

In order to further explore the absence of association between Neuroticism and cognitive decline observed in our cohort, an additional analysis was conducted to compare mean factors of Neuroticism of the whole study population $(n=590)$ with a reference population [39]. Neuroticism factor (and its facets Angry hostility, Depression and Vulnerability) scores were significantly lower in our series as compared to the group of reference (table 6). 
Table 4. Association between personality factors and facets, expressed as z-scores, and the risk of being in the dCON versus the sCON group

\begin{tabular}{|c|c|c|c|c|c|}
\hline \multirow[t]{2}{*}{ Personality traits } & & \multicolumn{2}{|l|}{ Crude } & \multicolumn{2}{|l|}{ Adjusted } \\
\hline & & OR $(95 \% \mathrm{CI})$ & $\mathrm{p}$ value & OR $(95 \% \mathrm{CI})$ & $\mathrm{p}$ value \\
\hline Neuroticism & $\mathrm{N}$ & $1.02(0.85,1.22)$ & 0.825 & $1.00(0.83,1.12)$ & 0.975 \\
\hline Anxiety & $\mathrm{N} 1$ & $1.06(0.88,1.27)$ & 0.542 & $1.06(0.88,1.28)$ & 0.526 \\
\hline Angry hostility & $\mathrm{N} 2$ & $1.04(0.87,1.24)$ & 0.695 & $1.03(0.85,1.23)$ & 0.793 \\
\hline Depression & N3 & $1.14(0.95,1.37)$ & 0.164 & $1.10(0.91,1.33)$ & 0.334 \\
\hline Self-consciousness & $\mathrm{N} 4$ & $0.93(0.77,1.11)$ & 0.404 & $0.91(0.75,1.09)$ & 0.307 \\
\hline Impulsiveness & N5 & $0.88(0.72,1.06)$ & 0.173 & $0.88(0.73,1.08)$ & 0.218 \\
\hline Vulnerability & N6 & $1.04(0.88,1.23)$ & 0.652 & $0.99(0.83,1.18)$ & 0.945 \\
\hline Extraversion & $\mathrm{E}$ & $0.87(0.73,1.04)$ & 0.135 & $0.89(0.74,1.07)$ & 0.212 \\
\hline Warmth & E1 & $0.93(0.78,1.11)$ & 0.446 & $0.93(0.77,1.11)$ & 0.416 \\
\hline Gregariousness & E2 & $0.89(0.75,1.07)$ & 0.226 & $0.91(0.75,1.09)$ & 0.303 \\
\hline Assertiveness & E3 & $0.97(0.82,1.15)$ & 0.727 & $0.99(0.83,1.19)$ & 0.939 \\
\hline Activity & E4 & $0.96(0.81,1.15)$ & 0.671 & $0.99(0.83,1.19)$ & 0.948 \\
\hline Excitement seeking & E5 & $0.91(0.76,1.09)$ & 0.292 & $0.89(0.74,1.07)$ & 0.214 \\
\hline Positive emotion & E6 & $0.86(0.72,1.02)$ & 0.088 & $0.87(0.72,1.05)$ & 0.147 \\
\hline Openness & 0 & $0.70(0.58,0.84)$ & $<0.001$ & $0.72(0.59,0.87)$ & 0.001 \\
\hline Fantasy & 01 & $0.84(0.69,1.02)$ & 0.074 & $0.85(0.70,1.04)$ & 0.106 \\
\hline Aesthetics & 02 & $0.78(0.65,0.93)$ & 0.006 & $0.77(0.64,0.93)$ & 0.008 \\
\hline Feelings & 03 & $0.81(0.67,0.97)$ & 0.023 & $0.82(0.68,1.00)$ & 0.045 \\
\hline Actions & 04 & $0.81(0.67,0.97)$ & 0.022 & $0.84(0.69,1.02)$ & 0.076 \\
\hline Ideas & 05 & $0.76(0.64,0.90)$ & 0.001 & $0.77(0.65,0.93)$ & 0.005 \\
\hline Values & 06 & $0.71(0.59,0.86)$ & $<0.001$ & $0.76(0.62,0.92)$ & 0.005 \\
\hline Agreeableness & A & $0.89(0.75,1.06)$ & 0.196 & $0.89(0.74,1.07)$ & 0.213 \\
\hline Trust & $\mathrm{A} 1$ & $0.85(0.71,1.01)$ & 0.065 & $0.85(0.71,1.02)$ & 0.087 \\
\hline Straightforwardness & $\mathrm{A} 2$ & $0.98(0.83,1.17)$ & 0.858 & $1.03(0.86,1.24)$ & 0.769 \\
\hline Altruism & A3 & $0.92(0.77,1.10)$ & 0.346 & $0.92(0.76,1.10)$ & 0.362 \\
\hline Compliance & $\mathrm{A} 4$ & $0.96(0.80,1.14)$ & 0.641 & $0.94(0.78,1.13)$ & 0.521 \\
\hline Modesty & A5 & $1.00(0.84,1.19)$ & 0.996 & $0.98(0.82,1.18)$ & 0.865 \\
\hline Tender-mindedness & A6 & $0.84(0.71,1.00)$ & 0.049 & $0.83(0.70,1.00)$ & 0.047 \\
\hline Conscientiousness & $\mathrm{C}$ & $1.13(0.94,1.35)$ & 0.205 & $1.14(0.94,1.38)$ & 0.173 \\
\hline Competence & $\mathrm{C} 1$ & $0.92(0.77,1.09)$ & 0.330 & $0.95(1.03,1.13)$ & 0.546 \\
\hline Order & $\mathrm{C} 2$ & $1.08(0.91,1.29)$ & 0.393 & $1.08(0.90,1.29)$ & 0.418 \\
\hline Dutifulness & $\mathrm{C} 3$ & $1.05(0.88,1.27)$ & 0.571 & $1.07(0.88,1.29)$ & 0.496 \\
\hline Achievement striving & $\mathrm{C} 4$ & $1.19(0.99,1.43)$ & 0.071 & $1.21(1.00,1.46)$ & 0.055 \\
\hline Self-discipline & $\mathrm{C} 5$ & $1.10(0.92,1.32)$ & 0.282 & $1.12(0.94,1.35)$ & 0.210 \\
\hline Deliberation & C6 & $1.21(1.00,1.46)$ & 0.049 & $1.19(0.98,1.44)$ & 0.090 \\
\hline
\end{tabular}

Total: $\mathrm{n}=488$; dCON: $\mathrm{n}=224$ (45.9\%). Logistic regression models adjusted for: sex, educational level and age at the time of cognitive assessment.

\section{Discussion}

Our findings in a community-based sample reveal that elderly controls with higher openness were less likely to experience subtle decline in cognitive function over an 18-month follow-up. Pointing to the relevance of these data in respect to AD progress, the same analysis in MCI compared to control cases led to similar associations in respect to personality factors and facets.

The association between personality 'Big Five' factors and facets and cognitive impairment is still a matter of debate. There is wide agreement that extraversion and agreeableness are 
Table 5. Association between personality factors and facets, expressed as z-scores, and the risk of being in the $\mathrm{MCI}$ versus the sCON group

\begin{tabular}{|c|c|c|c|c|c|}
\hline \multirow[t]{2}{*}{ Personality traits } & & \multicolumn{2}{|l|}{ Crude } & \multicolumn{2}{|l|}{ Adjusted } \\
\hline & & OR $(95 \% \mathrm{CI})$ & $\mathrm{p}$ value & OR $(95 \% \mathrm{CI})$ & $\mathrm{p}$ value \\
\hline Neuroticism & $\mathrm{N}$ & $1.05(0.84,1.32)$ & 0.660 & $1.12(0.88,1.42)$ & 0.350 \\
\hline Anxiety & N1 & $1.14(0.91,1.43)$ & 0.261 & $1.20(0.95,1.53)$ & 0.129 \\
\hline Angry hostility & $\mathrm{N} 2$ & $1.15(0.92,1.44)$ & 0.226 & $1.11(0.88,1.39)$ & 0.395 \\
\hline Depression & N3 & $1.01(0.80,1.28)$ & 0.908 & $1.09(085,1.39)$ & 0.493 \\
\hline Self-consciousness & $\mathrm{N} 4$ & $0.90(0.71,1.14)$ & 0.384 & $0.94(0.74,1.20)$ & 0.635 \\
\hline Impulsiveness & N5 & $0.82(0.64,1.05)$ & 0.110 & $0.85(0.66,1.09)$ & 0.191 \\
\hline Vulnerability & N6 & $1.07(0.86,1.32)$ & 0.535 & $1.17(0.93,1.46)$ & 0.175 \\
\hline Extraversion & $\mathrm{E}$ & $1.01(0.80,1.26)$ & 0.948 & $1.01(0.80,1.27)$ & 0.921 \\
\hline Warmth & E1 & $1.05(0.83,1.33)$ & 0.688 & $1.14(0.89,1.46)$ & 0.302 \\
\hline Gregariousness & E2 & $1.23(0.97,1.56)$ & 0.086 & $1.24(0.98,1.59)$ & 0.078 \\
\hline Assertiveness & E3 & $0.94(0.76,1.18)$ & 0.607 & $0.89(0.70,1.12)$ & 0.317 \\
\hline Activity & $\mathrm{E} 4$ & $0.80(0.64,1.01)$ & 0.063 & $0.79(0.62,1.01)$ & 0.059 \\
\hline Excitement seeking & E5 & $1.31(1.04,1.65)$ & 0.021 & $1.24(1.97,1.58)$ & 0.089 \\
\hline Positive emotion & E6 & $0.81(0.30,0.48)$ & 0.072 & $0.86(0.68,1.09)$ & 0.210 \\
\hline Openness & 0 & $0.55(0.43,0.71)$ & 0.000 & $0.61(0.46,0.79)$ & 0.000 \\
\hline Fantasy & 01 & $0.70(0.55,0.90)$ & 0.005 & $0.74(0.58,0.96)$ & 0.022 \\
\hline Aesthetics & 02 & $0.69(0.55,0.88)$ & 0.002 & $0.78(0.61,0.99)$ & 0.043 \\
\hline Feelings & 03 & $0.81(0.64,1.02)$ & 0.067 & $0.88(0.69,1.12)$ & 0.296 \\
\hline Actions & 04 & $0.59(0.46,0.75)$ & 0.000 & $0.63(0.49,0.81)$ & 0.000 \\
\hline Ideas & 05 & $0.63(0.50,0.80)$ & 0.000 & $0.63(0.49,0.81)$ & 0.000 \\
\hline Values & 06 & $0.61(0.48,0.77)$ & 0.000 & $0.64(0.50,0.83)$ & 0.001 \\
\hline Agreeableness & A & $0.84(0.67,1.05)$ & 0.120 & $0.91(0.72,1.16)$ & 0.467 \\
\hline Trust & $\mathrm{A} 1$ & $0.92(0.73,1.16)$ & 0.460 & $0.97(0.76,1.23)$ & 0.791 \\
\hline Straightforwardness & $\mathrm{A} 2$ & $0.87(0.70,1.09)$ & 0.229 & $0.96(0.76,1.22)$ & 0.739 \\
\hline Altruism & A3 & $0.94(0.75,1.19)$ & 0.623 & $1.02(0.80,1.29)$ & 0.892 \\
\hline Compliance & $\mathrm{A} 4$ & $0.84(0.67,1.05)$ & 0.130 & $0.89(0.70,1.13)$ & 0.328 \\
\hline Modesty & A5 & $1.03(0.82,1.31)$ & 0.784 & $1.07(0.84,1.37)$ & 0.578 \\
\hline Tender-mindedness & A6 & $0.76(0.61,0.96)$ & 0.020 & $0.80(0.63,1.02)$ & 0.069 \\
\hline Conscientiousness & $\mathrm{C}$ & $1.11(0.90,1.38)$ & 0.339 & $1.02(0.82,1.27)$ & 0.877 \\
\hline Competence & $\mathrm{C} 1$ & $0.94(0.75,1.17)$ & 0.568 & $0.86(0.68,1.10)$ & 0.227 \\
\hline Order & $\mathrm{C} 2$ & $1.24(0.98,1.58)$ & 0.069 & $1.20(0.94,1.52)$ & 0.146 \\
\hline Dutifulness & $\mathrm{C} 3$ & $0.99(0.80,1.22)$ & 0.910 & $0.96(0.77,1.19)$ & 0.687 \\
\hline Achievement striving & $\mathrm{C} 4$ & $1.27(1.00,1.61)$ & 0.050 & $1.18(0.92,1.51)$ & 0.188 \\
\hline Self-discipline & $\mathrm{C} 5$ & $1.17(0.93,1.48)$ & 0.182 & $1.10(0.87,1.39)$ & 0.434 \\
\hline Deliberation & C6 & $1.35(1.08,1.68)$ & 0.008 & $1.23(0.99,1.54)$ & 0.065 \\
\hline
\end{tabular}

Total: $\mathrm{n}=366$; MCI participants: $\mathrm{n}=102$ (27.87\%). Logistic regression models adjusted for sex, educational level and age at the time of cognitive assessment.

not related to the dementing process [18], a viewpoint that is further supported by the present results. In contrast, data on Neuroticism, Conscientiousness and Openness are less conclusive. Cross-sectional observations pointed to the presence of high levels of Neuroticism and low levels of consciousness both in AD [40] and MCI cases [41]. Previous longitudinal studies supported the association between higher levels of Neuroticism both in midlife and old age and dementia as well as MCI risk $[14,42,43]$. In contrast, higher conscientiousness conferred protection against dementia and $\mathrm{MCI}$, but this effect was marginal in most of the previous studies [18] and absent in the present series. More recent data challenged the association 
Table 6. Comparison of Neuroticism factor and fact scores between NEO-PI reference and the present series
Rodriguez et al.: Personality-Related Determinants of Subtle Cognitive Decline in Old Age: A Population-Based Study

\begin{tabular}{lllll}
\hline & & $\begin{array}{l}\text { NEO-PI } \\
\text { standards }\end{array}$ & $\begin{array}{l}\text { Current } \\
\text { sample }\end{array}$ & $\begin{array}{l}\mathrm{p} \\
\text { (t test) }\end{array}$ \\
\hline Neuroticism & $\mathrm{N}$ & $92.7 \pm 8.2$ & $76.6 \pm 18.6$ & $\mathbf{0 . 0 1 0}$ \\
Anxiety & $\mathrm{N} 1$ & $17.0 \pm 4.2$ & $13.8 \pm 5.2$ & 0.070 \\
Angry hostility & $\mathrm{N} 2$ & $16.2 \pm 3.0$ & $12.1 \pm 4.1$ & $\mathbf{0 . 0 0 3}$ \\
Depression & $\mathrm{N} 3$ & $15.9 \pm 3.0$ & $12.8 \pm 4.5$ & $\mathbf{0 . 0 3 9}$ \\
Self-consciousness & $\mathrm{N} 4$ & $15.0 \pm 3.0$ & $13.8 \pm 4.0$ & 0.381 \\
Impulsiveness & $\mathrm{N} 5$ & $15.6 \pm 2.9$ & $14.1 \pm 3.9$ & 0.276 \\
Vulnerability & $\mathrm{N} 6$ & $13.0 \pm 4.1$ & $9.9 \pm 4.1$ & $\mathbf{0 . 0 2 6}$ \\
\hline \multicolumn{2}{c}{ Values are presented as mean \pm SD. } \\
\hline
\end{tabular}

between high Neuroticism scores and dementia risk. In their 6-year longitudinal study based on the Dutch personality questionnaire, Ramakers et al. [13] showed that, although neuroticism scores showed negative correlation with mental speed in $\mathrm{MCI}$, there was no significant association between this variable and MCI conversion to AD.

Longitudinal studies addressing the association between NEO-PI patterns and cognitive decline in community-dwelling samples of healthy controls are still very rare. In a German population-based study including 222 elderly individuals followed up at three examination waves during a 10-year observation period [42], a decrease of neuroticism and extraversion scores over time was reported. A 2.24-higher risk of developing MCI at 10 years was observed in healthy controls with higher baseline neuroticism. In the same line, the Baltimore Epidemiologic Catchment Area study indicated a small but still significant negative association between neuroticism and MMSE scores. Higher levels of Consciousness were associated with a slight improvement in general cognitive function measures in this cohort [44]. Our data do not confirm the negative effect of higher Neuroticism levels on cognitive fate in normal aging. There are several explanations for this discrepancy. The German study had a significantly longer follow-up period focusing on the transition to MCI status. Since we focused our analysis on rapid cognitive changes, the length of our follow-up was of 18 months that allowed for observing subtle cognitive deficits at the pre-MCI stage. It is thus possible that, although higher Neuroticism scores increase the long-term risk for MCI transition, it does not impact on short-term cognitive changes in healthy controls. Unlike the present study, the Baltimore survey involved adult cases with a mean age of 45 at the time of personality assessment. A 10 -point increase in neuroticism score values was associated with only 0.15 -point decrease in MMSE score at the 10-year follow-up. Paralleling the findings of Ramakers et al. [13], the absence of difference in Neuroticism factor and facet scores also concerned the sCON/MCI comparison in our series. It is possible that the very low levels of Neuroticism observed in our sample (compared to age-adjusted normative data) reflect an over-representation of elderly persons with better mental health who are a priori less exposed to the deleterious effect of Neuroticism on cognition.

The most intriguing result of this study is the strong association between Openness factor levels and preservation of cognitive functions in elderly controls. In addition, Aesthetics, Feelings, Ideas and Values facet scores were all positively associated with sCON status at follow-up. Moreover, similar results for both Openness factor and facets were found when the distinction sCON/MCI was used as dependent variable in regression analyses. This observation goes beyond the weak positive effect reported in previous studies in MCI cohorts [18], implying that this personality parameter may have a protective effect on cognition that is mostly expressed in very early stages of cognitive impairment. Openness to experience refers 
Rodriguez et al.: Personality-Related Determinants of Subtle Cognitive Decline in Old Age: A Population-Based Study

to the breadth, depth, originality, and complexity of an individual's mental and experiential life. People with high scores in this factor (and corresponding facets) are more curious, open to changes, have richer internal lives and better coping abilities. Our findings suggest that these characteristics may represent a significant advantage to preserve cognitive performances in old age. Interestingly, higher openness to experience is also known to be associated with better quality of life both in younger non clinical samples and elderly cohorts [45-47].

Strengths of the present study include the large community-based sample, which may be seen as representative of the elderly population in the Geneva catchment area, exhaustive neuropsychological analysis at inclusion and follow-up, as well as careful exclusion of physical and mental conditions that may act as confounding factors in regression analyses. Some limitations of the present study are worth noting. The lack of a long prospective design poses some potential issues of directionality in the observed associations between personality traits and subtle cognitive impairment and causality cannot be implied. However, reverse causality (that is, subtle cognitive impairment leading to a biased over-reporting of openness) seems unlikely because there were no differences in the other four NEO-PI factors. Consistent with recent core clinical criteria for MCI [2], the identification of dCON was based on the objective decline in cognitive functions measured using serial, comprehensive neuropsychological assessments. However, in the absence of a longer follow-up, the cognitive fate of these cases remains uncertain so that they cannot be a priori considered as incipient AD cases. Although we adjusted for sociodemographic characteristics depressive and anxious symptomatology, the potential residual confounding effect of unmeasured lifestyles and health behaviors associated with both personality and cognitive impairment cannot be excluded [48]. When interpreting the present observations, one should keep in mind that multivariate regression models including the best predictors, openness facets, explain a mere $8 \%$ of the cognitive variability. The present analysis focuses on personality and does not take into account AD-related biomarkers (i.e. structural MRI parameters, amyloid burden, apolipoprotein E genotype) that could certainly increase the performance of our regression models. Future studies in community-based cohorts of healthy elders including longer follow-up periods, assessment of lifestyle measures and AD-related biomarkers are warranted to explore the relative contribution of personality in the prediction of cognitive decline.

\section{Acknowledgements}

This study was supported by grants SNF: 320030-129690/1 and SPUM 33CM30-124111 and JPND-BIOMARKAPD 31ND30_141625.

\section{References}

$>1$ Buckner RL: Memory and executive function in aging and AD: multiple factors that cause decline and reserve factors that compensate. Neuron 2004;44:195-208.

$\longrightarrow 2$ Albert MS, et al: The diagnosis of mild cognitive impairment due to Alzheimer's disease: recommendations from the National Institute on Aging-Alzheimer's Association workgroups on diagnostic guidelines for Alzheimer's disease. Alzheimers Dement 2011;7:270-279.

-3 Petersen RC, et al: Mild cognitive impairment: clinical characterization and outcome. Arch Neurol 1999;56: 303-308.

4 Caselli RJ, Reiman EM: Characterizing the preclinical stages of Alzheimer's disease and the prospect of presymptomatic intervention. J Alzheimers Dis 2013;33:S405.

5 Chételat G, et al: Relationships between brain metabolism decrease in normal aging and changes in structural and functional connectivity. Neuroimage 2013;76:167-177.

-6 Pietrzak RH, et al: Trajectories of memory decline in preclinical Alzheimer's disease: results from the Australian Imaging, Biomarkers and Lifestyle Flagship Study of Ageing. Neurobiol Aging 2015;36:1231-1238. 
7 Villemagne VL, et al: Comparison of $11 \mathrm{C}-\mathrm{PiB}$ and $18 \mathrm{~F}$-florbetaben for $\mathrm{A} \beta$ imaging in ageing and Alzheimer's disease. Eur J Nucl Med Mol Imaging 2012;39:983-989.

8 Mormino EC, et al: Amyloid and APOE epsilon4 interact to influence short-term decline in preclinical Alzheimer disease. Neurology 2014;82:1760-1767.

-9 Sutphen CL, et al: Longitudinal cerebrospinal fluid biomarker changes in preclinical Alzheimer disease during middle age. JAMA Neurol 2015;72:1029-1042.

10 Xekardaki A, et al: Arterial spin labeling may contribute to the prediction of cognitive deterioration in healthy elderly individuals. Radiology 2014;274:490-499.

11 Kumar R, et al: Clinical and neuroimaging correlates of mild cognitive impairment in a middle-aged community sample: the personality and total health through life 60+ study. Dement Geriatr Cogn Disord 2006;21:44-50.

12 Sargent-Cox K, et al: Subjective health and memory predictors of mild cognitive disorders and cognitive decline in ageing: the Personality and Total Health (PATH) through Life Study. Dement Geriatr Cogn Disord 2011;31:45-52.

$\checkmark 13$ Ramakers IH, et al: The effect of psychological distress and personality traits on cognitive performances and the risk of dementia in patients with mild cognitive impairment. J Alzheimers Dis 2015;46:805-812.

14 Terracciano A, et al: Personality and risk of Alzheimer's disease: new data and meta-analysis. Alzheimers Dement 2014;10:179-186.

15 Ausén B, et al: Personality features in subjective cognitive impairment and mild cognitive impairment-early indicators of dementia. Dement Geriatr Cogn Disord 2009;28:528-535.

16 Rolland J: Manuel de l'inventaire NEO-PI-R (Adaptation française) (Manual of the NEO-PI-R, French adaptation). Paris, ECPA, 1998.

17 Costa PT, McCrae RR: Four ways five factors are basic. Pers Ind Diff 1992;13:653-665.

18 Low L-F, Harrison F, Lackersteen SM: Does personality affect risk for dementia? A systematic review and meta-analysis. Am J Geriatr Psychiatry 2013;21:713-728.

19 Yen YC, et al: Cognitive impairment and associated risk factors among aged community members. Int J Geriatr Psychiatry 2004;19:564-569.

20 Bierman E, et al: Symptoms of anxiety and depression in the course of cognitive decline. Dement Geriatr Cogn Disord 2007;24:213-219.

-21 Folstein MF, Folstein SE, McHugh PR: 'Mini-mental state': a practical method for grading the cognitive state of patients for the clinician. J Psychiatr Res 1975;12:189-198.

22 Zigmond A, Snaith R: The hospital depression and anxiety scale. Acta Psychiatr Scand 1983;67:361-370.

23 Barberger-Gateau P, et al: Instrumental activities of daily living as a screening tool for cognitive impairment and dementia in elderly community dwellers. J Am Geriatr Soc 1992;40:1129-1134.

24 Wechsler D: Wechsler Adult Intelligence Scale. Third Edition (WAIS-III). San Antonio, Harcourt Assessment, 1997.

25 Reitan RM: Validity of the Trail Making Test as an indicator of organic brain damage. Percept Mot Skills 1958; 8:271-276.

26 Adam S, et al: L'épreuve de rappel indicé à 48 items (RI-48); in De Boeck H (ed): L'évaluation des troubles de la mémoire: présentation de quatre tests de mémoire épisodique (avec leur étalonnage). Marseille, Solal (Neuropsychologie), 2004, pp 49-67.

27 Van der Linden M, et al: L'épreuve de rappel libre/rappel indicé à 16 items (RL/RI-16). Marseille, Solal, 2004.

28 Baddeley AD, Emslie H, Nimmo-Smith I: The Doors and People Test: a test of visual and verbal recall and recognition. Bury St Edmunds, Thames Valley Test Company, 1994.

29 Heaton RK: A manual for the Wisconsin card sorting test. Western Psycological Services, 1981.

-30 Cardebat D, et al: Formal and semantic lexical evocation in normal subjects. Performance and dynamics of production as a function of sex, age and educational level (in French). Acta Neurol Belg 1990;90:207-217.

31 Kaplan E, Goodglass H, Weintraub S: The Boston Naming Test, ed 2. Philadelphia, Lea and Febiger, 1983.

-32 Ghent L: Perception of overlapping and embedded figures by children of different ages. Am J Psychol 1956;69: 575-587.

-33 Schnider A, et al: Ideomotor apraxia: behavioral dimensions and neuroanatomical basis. Brain Lang 1997;58: 125-136.

34 Poeck K: Clues to the nature of disruption to limb praxis; in Roy EA (ed): Neuropsychological Studies of Apraxia and Related Disorders. Amsterdam, Elsevier, 1985, pp 99-109.

35 Welsh KA, et al: The Consortium to Establish a Registry for Alzheimer's Disease (CERAD). Part V. A normative study of the neuropsychological battery. Neurology 1994;44:609-614.

-36 Hughes CP, et al: A new clinical scale for the staging of dementia. Br J Psychiatry 1982;140:566-572.

37 Petersen RC, et al: Current concepts in mild cognitive impairment. Arch Neurol 2001;58:1985-1992.

-38 Deiber M-P, et al: Attention and working memory-related EEG markers of subtle cognitive deterioration in healthy elderly individuals. J Alzheimers Dis 2015;47:335-349.

39 Costa PT Jr, McCrae RR: Revised NEO Personality Inventory (NEO-PI-R) and NEO Five-Factor Inventory (NEOFFI) Professional Manual. Odessa, Psychological Assessment Resources, 1992.

40 Robins Wahlin TB, Byrne GJ: Personality changes in Alzheimer's disease: a systematic review. Int J Geriatr Psychiatry 2011;26:1019-1029.

41 Donati A, et al: The evolution of personality in patients with mild cognitive impairment. Dement Geriatr Cogn Disord 2013;36:329-339. 
42 Kuzma E, et al: Premorbid personality traits and their course in mild cognitive impairment: results from a prospective population-based study in Germany. Dement Geriatr Cogn Disord 2011;32:171-177.

43 Johansson L, et al: Midlife personality and risk of Alzheimer disease and distress: a 38-year follow-up. Neurology 2014;83:1538-1544.

44 Hock RS, et al: Personality and cognitive decline in the Baltimore Epidemiologic Catchment Area follow-up study. Am J Geriatr Psychiatry 2014;22:917-925.

45 Bardi A, Ryff CD: Interactive effects of traits on adjustment to a life transition. J Pers 2007;75:955-984.

46 Steel P, Schmidt J, Shultz J: Refining the relationship between personality and subjective well-being. Psychol Bull 2008;134:138.

47 Weber K, et al: Personality, psychosocial and health-related predictors of quality of life in old age. Aging Ment Health 2015;19:151-158.

48 Norton S, et al: Potential for primary prevention of Alzheimer's disease: an analysis of population-based data. Lancet Neurol 2014;13:788-794. 